\section{FROM THE DECLINE AND FALL OF PROTEIN CHEMISTRY TO PROTEOMICS}

\section{Deb N. Chakravarti, PhD, DPhil (Oxon), Volume Editor}

\section{Computational Proteomics Supplement 32:S2-S3 (March 2002)}

In the fall of 2001, the editors of BioTechniques decided to launch a new quarterly Supplement Series to address the critical issues currently facing the biological research laboratory. It was more than fitting that they chose to devote the first edition to the emerging field of Computational Proteomics. The goal was to gather experts in the fields of bioinformatics and proteomics to explore the role of these technologies in drug discovery. The em phasis was to evaluate the bottleneck of the vast amount of protein data being generated and the different strategies that are being developed to overcome this bottleneck and accelerate the underlying process of identifying drug targets. It seems appropriate to introduce this publication with a brief historical background on the development of the science of proteomics followed by a short commentary on each article appearing in this issue.

In 1945, Fred Sanger developed one of the first methods for protein characterization by amino acid sequence analysis using fluorodinitrobenzene as part of his first Nobel prize winning work on the structure of insulin. A more efficient approach that used sequential degradation of residues from the amino terminus with phenylisothiocyanate was described in 1950 by Pehr Edman. Although a large number of protein sequences have been determined using automated "Edman degradation" over the years, the method is quite time-consuming and not amenable to large-scale identification of proteins and their primary structures. In the meantime, the advances in nucleic acid sequencing techniques and the ease of deduction of protein sequences from DNA sequences was reflected in an article published in Nature in 1978 entitled "The decline and fall of protein chemistry?" (4). As a young graduate student sequencing proteins under the supervision of the Nobel prize winning protein chemist Rodney Porter at the University of Oxford, I was obviously quite disappointed to read that. I did not have enough courage to ask him about the utility of becoming a protein chemist but posed the question to his $\mathrm{PhD}$ supervisor Fred Sanger. He assured me that the way developments were then taking place in recombinant DNA technology, in our time we should see advances in protein chemistry that had not even been contemplated at that time. He commented that we probably would be dealing with novel technology capable of characterizing proteins present in very trace amounts. I vividly remembered his words when fifteen years later, in 1995, it took me less than five minutes to analyze the tryptic digest of a protein band from a gel using a mass spectrometer and identify the protein by correlative sequence database searching. In most instances, that would now take less than a minute with the added luxury of a completely automated high-throughput process. The feasibility of such methods was first presented in a poster at the 1989 Protein Society Symposium in Seattle by W.J. Henzel, J.T. Stults, and C. Watanabe of Genentech and another one presented two years later at the Baltimore symposium by J.R. Yates, P.R. Griffin, T. Hunkapillar, S. Speicher, and L.E. Hood of Caltech (3). Since these contributions were not included in any published proceedings, the knowledge of the new method only gained lim ited circulation. It was not until 1993 that similar studies from five different groups appeared in the scientific literature.

The word proteome, indicating proteins expressed by a genome, was coined by Marc Wilkins and colleagues and first used in late 1994 at the Siena 2-D Electrophoresis meeting and appeared in published literature for the first time in July, 1995 (5). It is interesting to note that in the same month, the first complete sequence of the genome of a living organism, Haemophilus influenzae $\mathrm{Rd}$, was published. In the interim period, the complete sequence of several genomes has been determined, and the finished human genome will be available in the near future. However, our understanding of thousands to hundreds of thousands of proteins encoded by these genomes is lagging far behind. Since most of the cellular processes occur at the protein level, a thorough comprehension of the proteome, i.e., the pattern of proteins produced by a specific cell under a particular set of conditions, would definitely accelerate drug, vaccine, and diagnostic target discovery. Our current understanding of the human genome has led to the notion that there are fewer genes than originally thought, and this has become a strong driving force behind proteomics. Plans to elucidate the human proteome have been discussed at a meeting organized by the recently created Human Proteome Organization or HUPO (www.hupo.org) (1). In fact, the effort to produce an index of all human proteins, the human protein index, dates back to 1981 (2). The field of proteomics has rapidly expanded and includes diverse technologies like 2-D gel and mass spectrometry-based methods for protein profiling, protein microarrays, yeast twohybrid projects, protein-protein interaction pathways and cell signaling networks, high-throughput protein structural studies using mass spectrometry, nuclear magnetic resonance and X-ray crystallography, and high-throughput computational methods for protein 3-D structure as well as function determination.

Two-dimensional (2-D) gel electrophoresis and mass spectrometry based methods are currently the major experimental technologies for large-scale as well as high-throughput analysis of proteomes focused on protein identification and their qualitative and quantitative comparison. In the review article, Deb Chakravarti, Bulbul Chakravarti, and Ioannis Moutsatsos have addressed the state-of-the-art software and informatic tools for proteome profiling using these techniques. These authors have drawn attention to the lack of significant advances in the development of informatics and software tools to support the analysis and management of huge amounts of data generated in the process and integration of such information with other sources of protein structural and functional information. In this context the authors have discussed the importance of relational databases for protein profiling data management.

Proteome profiling is a very powerful tool in clinical medicine for identification of diagnostic markers. Clinical applications of proteomics can also provide information on drug targets, the mechanism of drug action, and drug-mediated toxicity. Eric Fung and Cynthia Enderwick have provided an elegant background of the ProteinChip technology based on the surface enhanced laser desorption/ionization time-of-flight (SELDI-TOF) 
mass spectrometry approach developed at Ciphergen Biosystems to determine protein expression patterns. Because of the wide dynamic range of protein expression in complex biological samples, initial sub-fractionation of the sample is carried out by retentate chromatography using ProteinChip arrays with differential surface chromatographic properties. These are then analyzed by matrix-assisted laser desorption/ionization time-of-flight (MALDITOF) mass spectrometry. The authors have provided a detailed discussion of the tools and strategies to manage and query the vast amounts of data generated in SELDI-TOF-based protein expression profiling projects to identify protein biomarkers.

A very important aspect of the functional proteomics approach involves the generation of large-scale protein-protein interaction networks. Vincent Schächter has described the informatics challenges related to the construction and prediction of such large-scale protein interaction maps. The article describes the construction of protein interaction networks based on experimental approaches involving the yeast two-hybrid system as well as computational predictive methods. The article describes in detail the reliability and validation of the interaction data generated by each approach. Finally, the author describes how the large amount of information contained in protein interaction maps can be utilized using visualization and exploration tools as well as functional annotation techniques. Analysis of the global structure of protein networks will lead to a better understanding of the cellular mechanisms with an evolutionary perspective.

The majority of drug target molecules are proteins and for a large number of proteins, 3-D structures are required for drug discovery. While high-throughput X-ray and NMR methods hold much promise for protein 3-D structure determination in the future, currently the only efficient avenue is to implement large-scale homology modeling programs to accompany structural proteomics initiatives. Kal Ramnarayan and his colleagues, Shankari Mylvaganam, Muthuchidambram Prabhakaran, Suresh Tudor, and Saied Moezzi have described their efforts on generation of large-scale, highly refined 3-D models of protein targets from their primary amino acid sequences using knowledge-based methods augmented by energy-based techniques, known as augmented homology modeling. In addition, these authors have described the organization of the structures determined using a relational database management system to allow extensive analytical processing and mining of the stored data.

Proteomics has presented the pharmaceutical industry with a sudden increase in the number of protein therapeutic targets available for drug development. One of the approaches for efficient drug discovery is to transform such validated targets into pharmaceutically relevant small molecule drug candidates using the tools of in silico design. Philip Dean and Edward Zanders have described the steps of the computational drug design process for exploiting protein structural information to generate virtual small molecules. These methods of structure-based and ligandbased design are particularly useful in handling the challenge of the large number of targets offered by proteomics.

Proteomics-based approaches to drug discovery platforms add another dimension of huge amounts of data generated from different instrumental methods. The protemics data need to be integrated with data from other sources like combinatorial chem istry programs, other high-throughput screening data, and pre-clinical as well as process or formulation research data, which are all part of the drug candidate selection and target validation process. John Helfrich discusses one solution to this problem using the NuGenesis Scientific Data Management System. The application-independent architecture of this system allows scientists to unify, catalog, share, and efficiently utilize data generated from diverse sources. The features and tools of this system in the context of drug discovery process have been discussed.

To extract information of value from proteomics for drug discovery and other purposes, it is necessary to build sophisticated data management systems and mining tools capable of manipulating and handling the huge amounts of information being generated in proteome projects. In the MarketView article, Enal Razvi discusses the potential market for informatic and computational tools for proteomics. This article also discusses market opportunities in various sub-areas of proteomics and some of the companies involved. As more and more big pharmaceutical concerns are focusing attention on the biotechnology and start-up companies for innovation, it will be interesting to watch the marketing strategies that will be developed by proteome informatics tool vendors.

In summary, the articles in this issue describe the major challenge of knowledge integration confronting proteome informatics: to combine and organize a massive amount of information from a wide range of experimental (e.g., 2-D gel, mass spectrometry, protein microarrays, yeast two-hybrid assays) and computational predictive methods with other sources of functional information (extracted from the scientific literature or structured databases) using appropriate knowledge management tools. Finally, I would like to emphasize that proteomics is not simple, many of the tools are in their infancy, and many more have not yet been defined.

[Note: This article was written by Deb N. Chakravarti, PhD, DPhil (Oxon), in his personal capacity as Volume Editor of the Computational Proteomics supplement to BioTechniques. The views expressed in this article do not necessarily represent the views of Wyeth Lederle Vaccines or Wyeth-Ayerst Research or the American Home Products Corporation.]

\section{ACKNOWLEDGMENTS}

I would like to acknowledge the untiring efforts and superb editorial skills of Christine Emerton, Manager of New Product Development for BioTechniques Press, for planning and bringing this issue out in a timely manner. I would also like to thank all the authors for their cooperation and striving for excellence.

\section{REFERENCES}

1.Abbott, A. 2001. Workshop prepares ground for human proteome project. Nature 413:763.

2.Anderson, N.G., A. Matheson, and N.L. Anderson. 2001. Back to the future: the human protein index (HPI) and the agenda for post-proteomic biology. Proteomics 1:3-12.

3.Cottrell, J.S. 1994. Protein identification by peptide mass fingerprinting Pept. Res. 7:115-124.

4.Malcolm, A.D. 1978. The decline and fall of protein chemistry? Nature 275:90-91.

5.Wasinger, V.C., S.J. Cordwell, A. Cerpa-Poljak, J.X. Yan, A.A. Gooley, M.R. Wilkins, M.W. Duncan, R. Harris, et al. 1995. Progress with gene-product mapping of the Mollicutes: Mycoplasma genitalium. Electrophoresis 16: 1090-1094. 